\title{
Performance analysis of coherent optical scatterometry
}

\author{
O. El Gawhary $\cdot$ N. Kumar · S.F. Pereira • \\ W.M.J. Coene · H.P. Urbach
}

Received: 11 April 2011 / Revised version: 18 August 2011 / Published online: 12 November 2011

(c) The Author(s) 2011. This article is published with open access at Springerlink.com

\begin{abstract}
Scatterometry is a well established technique currently utilized in research, as well as in industrial applications, to retrieve the properties of a given scatterer (the target) by looking at how the light coming from a certain source is diffracted in the far field. Currently the light source is often a discharge lamp that, after wavelength filtering, can be thought as a quasi-monochromatic, but spatially incoherent, source. In the present work, benefits of using a focused spot from a spatially coherent light source, as that emitted by a laser, are investigated on a theoretical viewpoint. The focused spot is scanned over the object of interest and, for each scan position, a far-field diffraction pattern is recorded. Our results show that spatially coherent light can sensibly increase the accuracy of the technique with respect to the target's geometrical profile.
\end{abstract}

\section{Introduction}

One of the oldest and most challenging problems faced in electromagnetism is represented by the requirement of retrieving some property of an object only looking at how an incident radiation is scattered by the object itself. The problem is interesting to investigate for many reasons. First

O. El Gawhary $(\bowtie) \cdot$ N. Kumar · S.F. Pereira $\cdot$ H.P. Urbach Optics Research Group, Department of Imaging Science and Technology, Delft University of Technology, P.O. Box 5046, 2600 GA Delft, The Netherlands

e-mail: o.elgawhary@tudelft.nl

H.P. Urbach

e-mail: h.p.urbach@tudelft.nl

W.M.J. Coene

ASML Netherlands B.V., De Run 6501, 5504 DR Veldhoven,

The Netherlands of all, several inspection methods are essentially based on this principle. For instance, we can recall here X-ray imaging techniques, optical coherent tomography for detection of human diseases or optical scatterometry for metrological applications, just to name a few [1,2]. These techniques are very intriguing also from a theoretical viewpoint, since they fall into the class of the so-called inverse problems and usually require special care in their treatment. The reason of that stems from the fact that they are nonlinear problems where the scattered radiation is measured putting some detector at a distance of several wavelengths from the scatterer in order to minimize eventual effects on the object itself. While this far-field regime decouples object and probe and actually simplifies the mathematical model, when compared to a near-field technique, it has however the drawback of turning the problem into an improperly-posed one. In fact, it was proven rigorously that any inverse technique in electromagnetism, that is based on far-field measurements, is severely ill-posed. This severe ill-posedness derives from the fact that there exists always an array of singular values of the operator that maps the near field of a radiating solution of Maxwell's equations to the corresponding far field, that approaches zero with arbitrary precision [3].

In order to stabilize the solution of a severely ill-posed problem like this, regularization methods have to be applied to achieve a good estimate of the object from the measured far field. In the present work we are mainly interested in optical scatterometry, that can be even more complicated. In fact, since the finite integration time of any detector is too large to resolve field oscillations at optical frequencies, only the field intensity is usually directly accessible. This means that the problem is not only to regularize the inversion from the far field to the object, but also that the far field is only partly known. 
Fig. 1 Grating profile with the corresponding shape parameters (midcd, swa, $h$ ) used to describe the geometrical profile

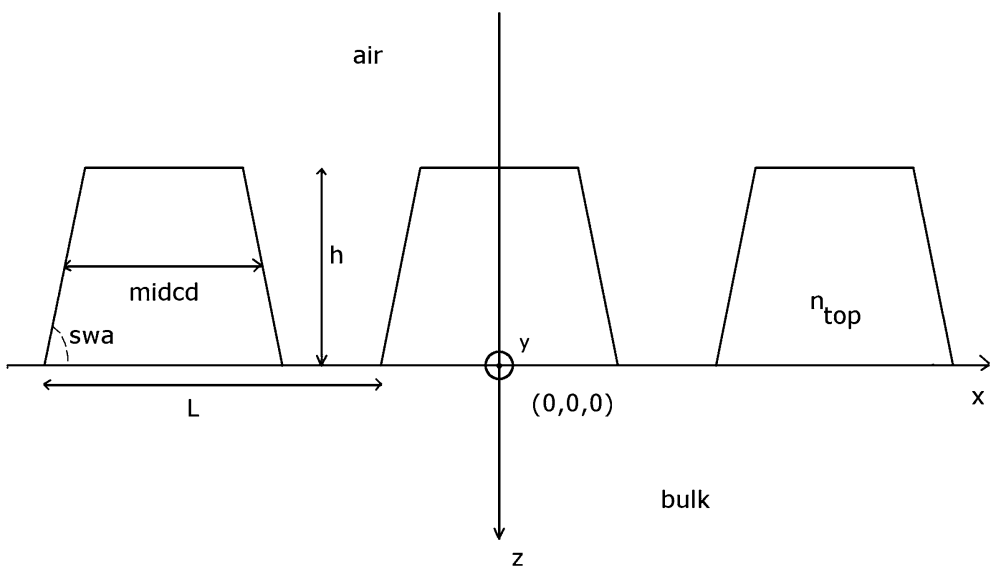

On the other hand, the importance of the technique in current industry processes is so high to deserve ad hoc studies in order to evaluate its real potentialities and limitations [4-8]. As an example, optical scatterometry is used in some applications of critical dimension (CD) metrology to determine with great precision (ranging from a few nanometers to even a fraction of a nanometer) the profile of a diffraction grating. The grating is usually described in terms of a finite numbers of parameters that have to be determined through measurements. The illuminating light is sent to the target and the scattered light is collected back to measure it either angularly or spectrally resolved. This spectrum is then compared to a simulated one, in order to find the set of parameters that minimizes the difference between measurements and simulations. The technique has several advantages: it is not affected by the diffraction limit, is fast and the implementation is not so technically demanding if compared to competing techniques [7]. Its use in the electronic industry is such that one can say that is the standard de facto. However, setting the limits and finding possible improvements is highly desirable to comply with current and near future industry's needs, as outlined in the ITRS roadmap of the semiconductors industry.

In the present paper, we evaluate whether and how the performance of optical scatterometry can be improved if spatially coherent light is utilized as source. The analysis is based on a comparison between the estimated uncertainty in the measurement of shape parameters when spatially coherent or completely incoherent light is used as illumination.

The paper is organized as follows. In Sect. 2, a model for the diffraction grating and the illumination is chosen, while in Sect. 3 we discuss the statistical model used to estimate the uncertainties on the shape parameters. In Sect. 4, the main results of the work are discussed and then a summary is presented in the Conclusions section.

\section{Models for grating and illumination}

Let us assume we have a perfectly periodic, one-dimensional, symmetric, infinite diffraction grating with spatial period $L$. By one-dimensional we mean that the grating properties are invariant along one direction (for example, the $y$-axis), while it is periodic along a perpendicular direction ( $x$-axis). For simplicity, and without a real loss of generality, we will consider a simple structure as that shown in Fig. 1. The grating consists of a bulk or substrate of index of refraction $n_{\text {bulk }}$ with, on top of it, a different medium of index of refraction $n_{\text {top. }}$ Generally speaking, these indices are complex-valued quantities. The surrounding medium is assumed to be air with $n=1$. Usually, the spatial period is known with good accuracy but not the actual shape within one period. This shape can be parameterized in terms of a given number of parameters representing the real unknowns that have to be determined by the measurements. In the present case, we consider a trapezoidal shape fully described through three parameters: side-wall angle (swa), critical dimension at half height (midcd) and height $(h)$, as shown in Fig. 1.

Additionally, one has to specify the properties of the illumination. A simple sketch is shown in Fig. 2. The light coming from the source passes through a high-NA objective and is subsequently focused onto the grating (NA, for numerical aperture). After interacting with the grating, part of the scattered light is collected again by the same objective and directed to the detector (not indicated in the figure). The advantage of using an objective lens resides in the fact that it is possible to illuminate the grating with different incident plane waves at the same time (all those plane waves that lie within the NA). Considering that the objective is usually illuminated by an expanded beam, we will assume, from now on, that in the back focal plane of the objective there is a uniform field distribution and that the role of the lens is only to convert the light coming from each point of that plane in a plane wave impinging on the grating itself. 
Fig. 2 Simple sketch showing how the incoming light is incident on the grating through an objective lens of numerical aperture NA. The gratings dimensions are not in scale for clarity reasons. The zoomed area simply shows the typical structure of a focused field

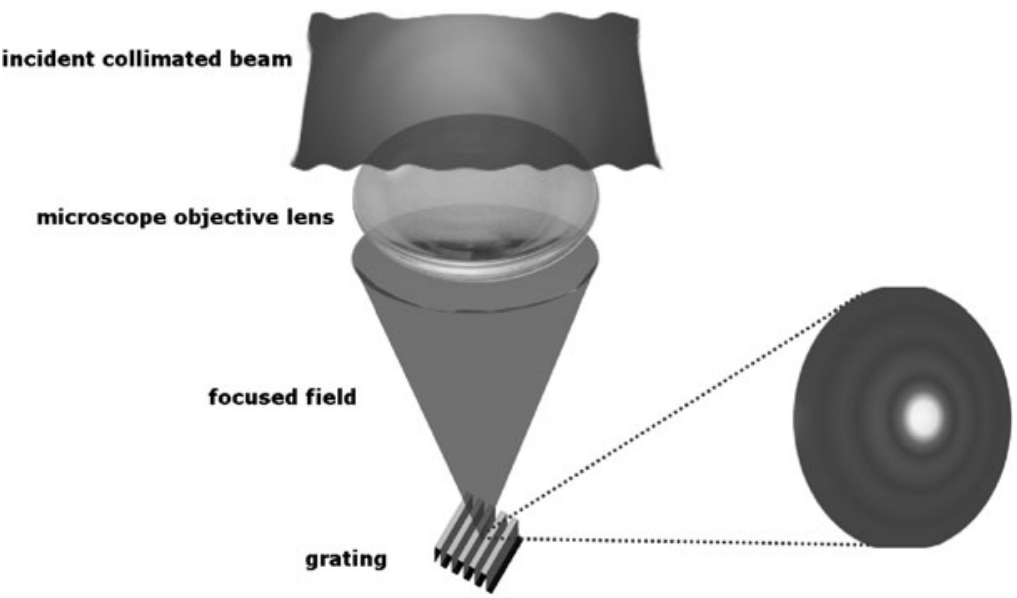

Also, for simplicity it will be assumed, from now on, that $\sigma_{i}=\sigma, \forall i$. It is reasonable to say that when the set of shape parameters coincides, within some accuracy, with the real one, then the distance between measurements and simulations should be minimal. In statistical theory, a good way to define such a distance is using the $\chi^{2}$ distribution

$\chi^{2}=\frac{1}{N} \sum_{i=1}^{N}\left[\frac{\mathrm{I}_{i}^{(m)}-\mathrm{I}\left(\mathbf{p}_{i}, \mathbf{a}\right)}{\sigma_{i}}\right]^{2}$

and to look for that set of parameters $\mathbf{a}_{\min }$ that minimizes it [14]. Of course, in case of an ideal noiseless system (i.e. $\left.\sigma_{i}=0, \forall i\right)$, and the assumption that the mathematical model is complete, the minimum of $\chi^{2}$ would lead exactly to the right set of parameters without any uncertainty. However, some noise will always be present and this raises the issue of how good, or accurate, a parameter estimation is.

What is interesting in this approach is that all estimated uncertainties, $\Delta \mathbf{a}$ say, on the shape parameters can be derived by expanding (1) in Taylor's series around the minimum. The second order derivative of such expansion is:

$$
\begin{aligned}
\frac{\partial^{2} \chi^{2}}{\partial a_{j} \partial a_{k}}= & \frac{2}{N} \sum_{i=1}^{N} \frac{1}{\sigma_{i}^{2}}\left[\frac{\partial \mathrm{I}\left(\mathbf{p}_{i}, \mathbf{a}\right)}{\partial a_{j}} \frac{\partial \mathrm{I}\left(\mathbf{p}_{i}, \mathbf{a}\right)}{\partial a_{k}}\right. \\
& \left.-\left[\mathrm{I}_{i}^{(m)}-\mathrm{I}\left(\mathbf{p}_{i}, \mathbf{a}\right)\right] \frac{\partial^{2} \mathrm{I}\left(\mathbf{p}_{i}, \mathbf{a}\right)}{\partial a_{k} \partial a_{j}}\right] .
\end{aligned}
$$

In (2) the indexes $j$ and $k$ refer to the shape parameters. Usually, the term containing the second-order derivative in (2) is neglected, since summing on a large number of points $N$ tends to average the difference $\left[\mathrm{I}_{i}^{(m)}-\mathrm{I}\left(\mathbf{p}_{i}, \mathbf{a}\right)\right]$ out. This assumption leads to a matrix $\left\{\alpha_{j k}\right\}$

$\left[\alpha_{j k}\right]=\frac{1}{2} \frac{\partial^{2} \chi^{2}}{\partial a_{k} \partial a_{l}}=\frac{1}{N} \sum_{i=1}^{N} \frac{1}{\sigma_{i}^{2}}\left[\frac{\partial \mathrm{I}\left(\mathbf{p}_{i}, \mathbf{a}\right)}{\partial a_{j}} \frac{\partial \mathrm{I}\left(\mathbf{p}_{i}, \mathbf{a}\right)}{\partial a_{k}}\right]_{\mathbf{a}=\mathbf{a}_{\min }}$,

with $j, k=1,2,3, \ldots$, 
that is related to the estimated 3-sigma uncertainties for the shape parameters $\mathbf{a}$ as in the following:

$\Delta a_{j}=3 \sqrt{C_{j j}}$,

where $\left[C_{j k}\right]=\left[\alpha_{j k}\right]^{-1}$ (again, for details, the reader can refer to [12]). An analysis of these uncertainties is fundamental to check how good the whole system is. In fact, large uncertainties mean that the technique does not resolve large variations of the shape parameters, which in turn denotes very low sensitivity with respect to the grating's profile. Stated differently, large uncertainties imply that several realizations of the set $\mathbf{a}$ are equally compatible with the measurements and the technique cannot discern among them. On the other hand, small uncertainties are an indication that good accuracy in determining the shape parameters can be reached. It follows that a comparison between the corresponding estimated uncertainties on the shape parameters is a way to determine whether a chosen technique is better than another.

In the next section we are going to make such a comparison, when coherent or incoherent light is used as illumination, by discussing a few examples.

\section{A comparison between spatially coherent and spatially incoherent light}

Once the model for the grating and for the estimated uncertainties on its shape parameters is available, one is ready to analyze the effect of coherence. Before going into the details of the present analysis, we need to clarify one aspect. When monochromatic, spatially incoherent light is used as a source, a diffraction-limited spot cannot be generated. This implies that a large spot illuminates a large portion of the grating at once (as compared with spatially coherent illumination). However, if spatially coherent illumination of same wavelength is used, then the spot size can be of the order of the wavelength. It follows that if a strongly focused coherent illumination is applied, in order to cover the grating, a scanning scheme has to be implemented. Different scanning strategies can be realized. In our case, we will suppose that during scanning of the object there is always a good overlap between two adjacent positions. This was recently profitably used in the context of phase retrieval problems where it resulted to be fundamental to reconstruct the original object's amplitude and phase from a collection of far-field intensity measurements [15-17]. In order to make a fair comparison, one should also take into account that since scanning adds more additional data points, and recalling that the final uncertainties scale with the total number $N$ of data points as $1 / \sqrt{N}$, more points imply smaller uncertainties. To be more precise, if only one position is considered in case of incoherent illumination and $M$ positions for the coherent one, then in the latter case each uncertainty has to be increased by multiplying by a $\sqrt{M}$ factor. This is the reason that we have defined the $\chi^{2}$ distribution in (1) in a slightly different way, with respect to the standard form found in literature, by dividing by the number of points $N$. In this way we have removed any difference in the uncertainties only originating from having a different number of data points.

The need for scanning has further consequences. On an experimental viewpoint it makes obviously the measurement process slower, since more acquisition steps are required. On the other hand, it also affects the uncertainties estimation analysis, as presented here. In fact, at every new position it is necessary to solve rigorously the scattering problem between incident field and grating, which is time consuming. Fortunately, in case of an ideal infinite periodic grating the complex amplitude $\mathbf{R}_{n}$ of the $n$th diffraction order in any shifted position can be derived easily from the same original position just by adjusting the phase. For reader's convenience, the expression of the correct phase correction is reported in the Appendix along with a short derivation.

Moving now to a concrete example, let us consider a grating, as shown in Fig. 1, with $L=189 \mathrm{~nm}, \operatorname{midcd}=27 \mathrm{~nm}$, $s w a=87^{\circ}, h=40 \mathrm{~nm}$ and NA $=0.95$. We choose silicon as bulk material and polymethyl methacrylate (PMMA) resist as grating material. We study the uncertainties on a range of possible wavelengths in the interval $\lambda \in(145 \mathrm{~nm}, 450 \mathrm{~nm})$. In order to build the matrix $\left[\alpha_{j k}\right]$ in (3) one has to implement the derivatives numerically. In the present case, a central difference formula is used with an increment of $0.1 \mathrm{~nm}$ in case of midcd and height variation and $0.1^{\circ}$ in case of swa variation.

In the case of coherent illumination, three different positions were considered. An explicative plot is reported in Fig. 3 (where only two positions are shown). In the subplot (a) the incident spot is initially supposed to be centered on one line. The other two positions (subplot (b)) correspond to the same lateral shift $\Delta x$, but in two opposite directions. Since the absolute value of such uncertainties depends on the noise level considered (i.e. on the $\left\{\sigma_{i}\right\}$ introduced in the previous section) as well as on the number of points $N$ in the detector, it is somehow advantageous to discuss the results independently of these values that can change from case to case. For this reason, in Fig. 4 the ratios between the uncertainties $\Delta a^{\text {inc }}$ and $\Delta a^{\text {coh }}$ (see (4)) for incoherent light and those for coherent case are shown for the three parameters of interest.

All plots are shown as function of the dimensionless variable $\lambda /($ NA $L$ ). This is convenient since it allows us to check easily how many diffraction orders enter into the exit pupil. In fact, when $\lambda /(\mathrm{NA} L) \geq 2$, only the scattered zerothorders of all incident plane waves fall into the NA of the microscope objective. Since coherence can manifest itself 

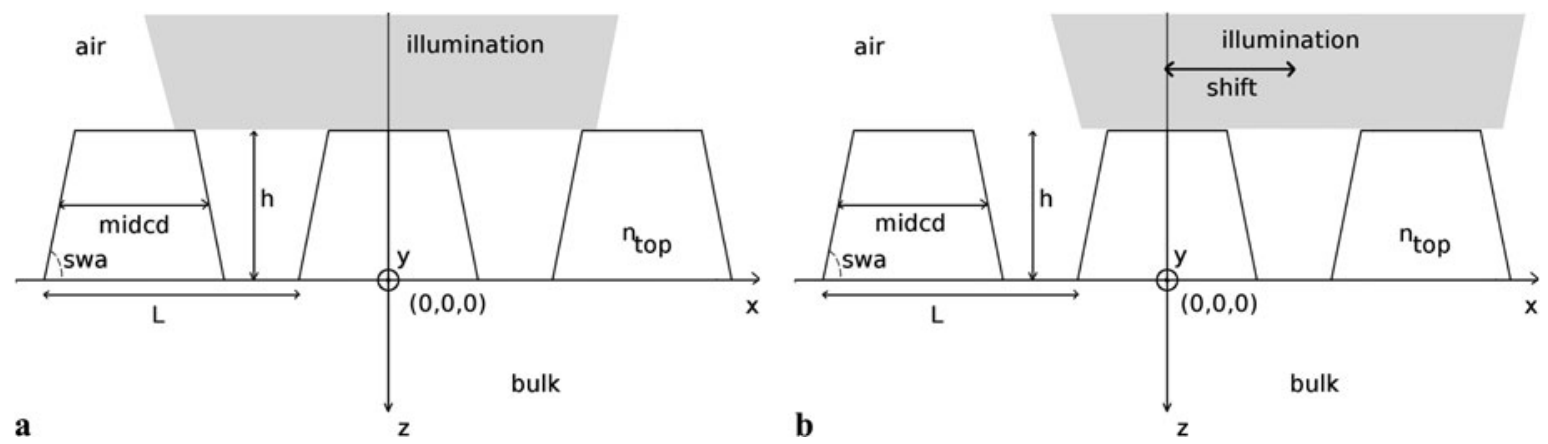

Fig. 3 Initial (subfigure (a)) and one shifted configuration (subfigure (b), shift $\Delta x$ ) in case of coherent illumination
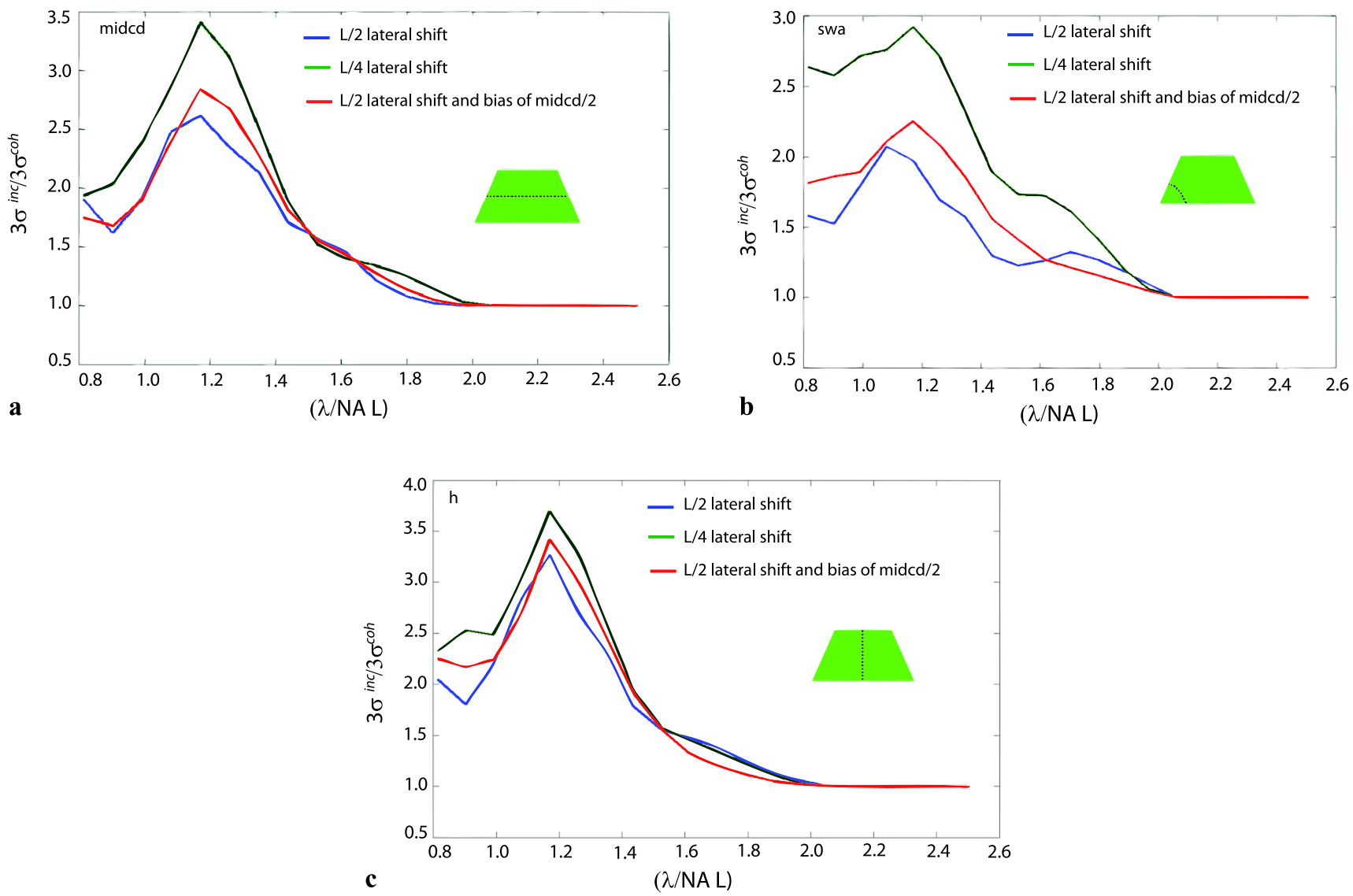

Fig. 4 Ratio between the uncertainties $\Delta a^{\text {inc }}$ and $\Delta a^{\text {coh }}$, in case of incoherent and coherent light respectively. $L=189 \mathrm{~nm}$ is the grating's period and $\lambda$ the wavelength. NA $=0.95$ is the numerical aperture. The

only in terms of interference effects between overlapping orders, in that case coherent and incoherent light behave in the same way and eventual coherent effects cannot be seen. When $1 \leq \lambda /($ NA $L)<2$, also the first orders are included in the lens pupil. In general, when $\lambda /(\mathrm{NA} L)<2 / s$, with $s$ positive integer, then the first $s$ th orders are collected by the lens.

To analyze also the dependence on the lateral shift, we have studied different situations. All black lines in Fig. 4 curves were obtained by varying $\lambda$ in the range of $(145 \mathrm{~nm}, 450 \mathrm{~nm})$. (a) midcd, (b) swa, (c) $h$. It should be noted that the dispersion relation for the optical constants $n$ and $k$ is accounted for correspond to a lateral shift $\Delta x=L / 2$. The green ones were computed with a shift of $L / 4$ while the red ones again with a lateral shift of $L / 2$ but including also an initial bias of midcd $/ 2$. The reason for introducing such bias is twofold and will be clearer later on.

If we look at Fig. 4(a), black line, we see that for large values of $\lambda /(\mathrm{NA} L)$ (larger than 2 ) the ratio between the two uncertainties is unity. This means that there is no difference between the two configurations, as it should be. How- 
ever, as soon as $\lambda /(\mathrm{NA} L)$ tends to be smaller than 2 , then some difference shows up. The first orders start to be collected by the lens and, as a consequence of that, the uncertainty ratio departs from unity. It gets bigger than one in the whole range of studied wavelengths reaching its maximum for $\lambda /(\mathrm{NA} L)=1.17$, where the uncertainty obtained using coherent light is more that 2.5 smaller than that obtainable using incoherent light. It is also important to notice that the ratio is not a monotone function of the independent variable $\lambda /(\mathrm{NA} L)$. Even more important is to observe that when the lateral shift is smaller, as in the green line (shift $L / 4$ ), the difference between the two cases gets even bigger. In that case the maximum gain is about 3.4. To understand this phenomenon, one should recall that the focused field does not have a uniform amplitude but shows an Airy pattern-like structure. Intuitively, we can say that the field is more sensitive to shape changes when the central lobe is in the vicinity of one line and/or its edges. This is not the case for a shifted beam when the beam's spot size is small enough and the period $L$ and the lateral shift $\Delta x$ are both much larger than the midcd. As it has been mentioned before, in order to confirm this effect, we introduced a lateral shift (bias) between the relative position grating-spot and we computed the uncertainty ratio for the case when the lateral shift is again $L / 2$ but the incident beam is initially centered on one line's edge (Fig. 4(c), red curve). As expected, the ratio is now higher. This proves that using optical coherent scatterometry provides also information on the relative spatial position between illumination and grating.

The same analysis can be repeated for the other two parameters, swa and height. In Fig. 4(b), the uncertainty ratios for swa are presented with essentially the same features discussed before. At last, Fig. 4(c) shows the uncertainty ratio for the height. Here an additional comment is necessary. It is manifest that the difference among the three scanning schemes is now notably reduced. But this is not surprising, since the detection of height variations is expected to be less sensitive to the relative position grating-illumination than changes in the midcd and swa.

For what we said so far, it follows that a coherent optical scatterometer can lead to benefits only when at least part of the first diffracted orders lie into the exit pupil. This fixes some constraint between the wavelength $\lambda$ and the pitch $L$ and, consequently, on the scanning scheme as well. In fact, one has to scan one period of the grating by keeping some overlap between adjacent spots, which has consequences on the minimum number of scanning positions necessary within one period. Once $\lambda$ and NA are fixed, the minimum pitch to measure the first diffracted orders is $L_{\min }=\lambda /(2 \mathrm{NA})$. On the other hand, the spot size can be estimated, using Airy's expression, as $r_{\text {spot }}=0.61 \lambda /$ NA. This implies that $r_{\text {spot }} / L_{\min }=1.22$, and it represents a constraint for the distance between two consecutive adjacent spots, considered that the lateral shift is a fraction of $r_{\text {spot }}$.

\section{Conclusions}

To summarize, a theoretical analysis on a spatially coherent optical scatterometry has been described. After considering a specific model for the grating and for the illumination path, we computed the expected uncertainties in the shape parameters under the assumption of Gaussian distribution for the measurement noise and for the wavelength in the range $(145 \mathrm{~nm}, 450 \mathrm{~nm})$. We have shown that, once a proper strategy on how to scan the sample is chosen, spatial coherence leads to an increase in the accuracy of the grating's profile reconstruction. Compared with the incoherent case, it was shown that in some circumstances the estimated uncertainties on the shape parameters can be up to more than three times smaller than those obtained using a standard discharge lamp.

Acknowledgements The Authors acknowledge Mark van Kraaij for providing the RCWA code and Irwan Setija, Stefan Petra and Arie den Boef for extensive discussions. O. El Gawhary and N. Kumar acknowledge the Surface Physics for Advanced Manufacturing European project, within the FP7 Marie Curie Research and Initial Training Network Program, for funding the research under the Project Number 215723.

Open Access This article is distributed under the terms of the Creative Commons Attribution Noncommercial License which permits any noncommercial use, distribution, and reproduction in any medium, provided the original author(s) and source are credited.

\section{Appendix: How to include the lateral shift into a rigorous electromagnetic code without running additional simulations}

In Sect. 4 we stated that it is not necessary to re-run an additional simulation, after shifting the illumination of an amount $\Delta x$, to compute the complex amplitude of all diffraction orders, once the result in the original configuration (i.e. without any shift) is available. The aim of this Appendix is to derive the expression of the phase shift that is needed to do that.

Let us assume that an incident field $\mathbf{E}^{\text {inc }}(x, y, z)$ impinges on an infinite grating that is invariant along the $y$ direction. $\mathbf{E}^{\text {inc }}(x, y, z)$ can be any general field, but we will focus only on one of the plane waves, $\mathbf{u} \exp \left[i\left(k_{x} x+k_{y} y+\right.\right.$ $\left.k_{z} z\right)$ ], representing its angular spectrum. As usual, $k_{x}, k_{y}, k_{z}$ are the components of the wave vector of the incident wave and $\mathbf{u}$ is the unit vector describing the polarization state. The total field above the grating can be written then in terms of the Rayleigh expansion as [18, 19]

$$
\begin{aligned}
\mathbf{E}_{1}(x, y, z)= & \sum_{n} \mathbf{R}_{n} \exp \left[i\left(k_{x n} x+k_{y n} y-k_{z n} z\right)\right] \\
& +\mathbf{u} \exp \left[i\left(k_{x} x+k_{y} y+k_{z} z\right)\right]
\end{aligned}
$$


where $k_{x n}, k_{y n}$ and $k_{z n}$ are the components of the wavevector of the $n$th plane wave, with $k_{x n}^{2}+k_{y n}^{2}+k_{z n}^{2}=k^{2}$, with $k=2 \pi / \lambda$. From Bragg's law we know that $k_{x n}, k_{y n}$ and $k_{z n}$ are related to $k_{x}, k_{y}$ and $k_{z}$ through the relations

$k_{x n}=k_{x}+\frac{2 \pi n}{L}$

$k_{y n}=k_{y}$

$k_{z n}=\sqrt{k^{2}-k_{x n}^{2}-k_{y n}^{2}}$

with $L$ the period of the grating. The complex amplitudes $\mathbf{R}_{n}$ are the unknowns that have to be determined by solving Maxwell's equations.

If we now shift the whole (5) by $\Delta x$ along the $x$ direction, we have

$$
\begin{aligned}
& \mathbf{E}_{1}(x-\Delta x, y, z) \\
& =\sum_{n} \tilde{\mathbf{R}}_{n} \exp \left[i\left(k_{x n}(x-\Delta x)+k_{y} y-k_{1, z n} z\right)\right] \\
& \quad+\exp \left[i\left(k_{x}(x-\Delta x)+k_{y} y+k_{z} z\right)\right] \mathbf{u} \\
& =\sum_{n} \tilde{\mathbf{R}}_{n} \exp \left(-i k_{x n} \Delta x\right) \exp \left[i\left(k_{x n} x+k_{y} y-k_{1, z n} z\right)\right] \\
& \quad+\exp \left[i\left(k_{x} x+k_{y} y+k_{z} z\right)\right] \exp \left(-i k_{x} \Delta x\right) \mathbf{u},
\end{aligned}
$$

where $\tilde{\mathbf{R}}_{n}$ are the new coefficients that have to be computed in the new configuration. Now from (7) we see that the incident plane wave propagates exactly along the same direction, which means that the incident wave vector is unchanged. Hence the interaction with the infinite grating, which is a linear process, is the same as before. The only difference consists in having a different complex amplitude, so that the final effect of shifting should be the presence of a constant term $\exp \left[-i k_{x}(\Delta x)\right]$ in front of the scattered field. In other words, the final result must be

$$
\begin{aligned}
& \mathbf{E}_{1}(x-\Delta x, y, z) \\
& =\sum_{n} \exp \left(-i k_{x} \Delta x\right) \mathbf{R}_{n} \exp \left[i\left(k_{x n} x+k_{y} y-k_{1, z n} z\right)\right] \\
& \quad+\exp \left[i\left(k_{x} x+k_{y} y+k_{z} z\right)\right] \exp \left(-i k_{x} \Delta x\right) \mathbf{u} .
\end{aligned}
$$

It is possible to get (8) from (7) under the condition

$$
\exp \left(-i k_{x} \Delta x\right) \mathbf{R}_{n}=\tilde{\mathbf{R}}_{n} \exp \left(-i k_{x n} \Delta x\right)
$$

which in turn implies, from (6),

$\tilde{\mathbf{R}}_{n}=\mathbf{R}_{n} \exp (i 2 \pi n / L \Delta x)$.

This represents the phase correction we were looking for to account for the lateral shift $\Delta x$.

\section{References}

1. International Technology Roadmap for Semiconductors, Metrology, ITRS Web Resources. http://www.itrs.net/Links/2009ITRS/ 2009Chapters_2009Tables/2009_Metrology.pdf (2009)

2. M. Born, E. Wolf, Principles of Optics, 7th edn. (Cambridge University Press, Cambridge, 2001), pp. 217-227

3. D. Colton, R. Kress, Inverse Acoustic and Electromagnetic Scattering Theory, 2nd edn. (Springer, Heidelberg, 1998), pp. 85-93

4. American National Institute of Standards and Technology, Optical Grating Scatterometry. http://www.nist.gov/physlab/div844/ grp06/scatterometry.cfm

5. E. Barouch, S.L. Knodle, Proc. SPIE 5038, 559567 (2003)

6. C. Ko, Y. Ku, Opt. Express 14, 6001 (2006)

7. M. Wurm, B. Bodermann, "Non-imaging optical metrology of structured surfaces: Prospects, challenges, and limitations", presented at the Seminar on Scatterometry and Ellipsometry on structured surfaces, Braunschweig, Germany, 18-19 March 2009

8. I. Gereige, S. Robert, S. Thiria, F. Badran, G. Granet, J.J. Rousseau, J. Opt. Soc. Am. A 25, 1661 (2008)

9. M.G. Moharam, T.K. Gaylord, J. Opt. Soc. Am. 71, 811 (1981)

10. M.G. Moharam, E.B. Grann, D.A. Pommet, J. Opt. Soc. Am. A 12, $1068(1995)$

11. L. Li, J. Opt. Soc. Am. A 13, 1870 (1996)

12. W.H. Press, S.A. Teukolsky, W. Vetterling, B.P. Flannery, Numerical Recipes in C, The Art of Scientific Computing, 2nd edn. (Cambridge University Press, Cambridge, 1995), pp. 657-674

13. R. Silver, T. Germer, R. Attota, B.M. Barnes, B. Bunday, J. Allgair, E. Marx, J. Jun, Proc. SPIE 6518, 65180U (2007) (17 pp.)

14. A. Papoulis, Probability, Random Variables and Stochastic Processes (McGraw-Hill, New York, 1991), Chapter 9

15. J.M. Rodenburg, A.C. Hurst, A.G. Cullis, Ultramicroscopy 107, 227 (2007)

16. H.M.L. Faulkner, J.M. Rodenburg, Ultramicroscopy 103, 153 (2005)

17. J.M. Rodenburg, A.C. Hurst, A.G. Cullis, B.R. Dobson, F. Pfeiffer, O. Bunk, C. David, K. Jefimovs, I. Johnson, Phys. Rev. Lett. 98, 034801 (2007)

18. L. Rayleigh, Proc. R. Soc. Lond. Ser. A 79, 399 (1907)

19. A.V. Tishchenko, Opt. Express 17, 17102 (2009) 